\title{
DESARROLLO Y VALIDACIÓN DEL MÉTODO DE AMPLIFICACIÓN ISOTÉRMICA MEDIADA EN LAZO PARA LA DETECCIÓN DEL VIRUS ZIKA
}

\author{
Oscar Escalante-Maldonado, ,a, Ronnie Gustavo Gavilán 1,a , Maria Paquita García ${ }^{1, b}$, Adolfo Marcelo ${ }^{1, a}$, \\ Edson Pacheco ${ }^{1, a}$, César Cabezas ${ }^{1, c}$, Wataru Yamazaki ${ }^{2, a}$
}

\begin{abstract}
RESUMEN
Se desarrolló un método de amplificación isotérmica mediada en lazo de transcriptasa inversa (RT-LAMP) para detectar Zika. Los primers se diseñaron basándose en la región NS5 de 64 genomas completos. Se usó reactivo LAMP liofilizado. Inicialmente, se probaron siete arbovirus diferentes y solo las muestras de Zika resultaron positivas. Además, las diluciones seriadas de una de los ARN de Zika se compararon mediante RT-LAMP y qRT-PCR, demostrando que RTLAMP es 1000 veces más sensible. También se evaluó 300 muestras de suero usando RT-LAMP y los resultados se compararon con los métodos de qRT-PCR estándar y obtuvimos un 99,3\% (IC95\%: 97,7 - 100,0) de sensibilidad, 100\% (IC95\%: 99,7 - 100,0) de especificidad, 100\% (IC95\%: 99,7 -100,0) de valor predictivo positivo y 99,3\% (IC95\%: 97,7 $100,0)$ de valor predictivo negativo. En conclusión, este método brinda una alternativa de bajo costo, alto rendimiento, viabilidad y confiabilidad para el diagnóstico rápido de Zika en instalaciones de atención primaria de salud.
\end{abstract}

Palabras clave: Virus Zika; Diagnóstico; ADN polimerasa dirigida por ARN; Reacción en cadena de la polimerasa. (Fuente: DeCS BIREME).

\section{DEVELOPMENT AND VALIDATION OF LOOP-MEDIATED ISOTHERMAL AMPLIFICATION FOR THE DETECTION OF THE ZIKA VIRUS}

\begin{abstract}
A Reverse Transcription Loop-mediated Isothermal Amplification (RT-LAMP) method was developed to detect Zika. The primers were designed based on the NS5 region of 64 complete genomes. Lyophilized LAMP reagent was used. Initially, seven different arboviruses were tested and only Zika samples tested positive. Additionally, serial dilutions of one of Zika's RNA were compared using RT-LAMP and qRT-PCR, demonstrating that RT-LAMP is 1,000 times more sensitive. We also evaluated 300 serum samples with RT-LAMP comparing the results with standard qRT-PCR methods, and we obtained a $99.3 \%$ sensitivity, $100 \%$ specificity, $100 \%$ positive predictive value, and $99.3 \%$ negative predictive value. In conclusion, this method provides a low-cost, high-performance, viable, and reliable alternative for the rapid diagnosis of Zika in primary health-care facilities.
\end{abstract}

Keywords: Zika virus; Diagnosis; RNA-directed DNA polymerase; Polymerase chain reaction (source: MeSH NLM).

\section{INTRODUCCIÓN}

El Zika es una enfermedad febril causada por un flavivirus denominado el virus Zika (ZIKV) ${ }^{(1)}$. La capacidad de este virus para adaptarse a nuevos vectores ${ }^{(2)}$ y la influencia de otros factores tales como el cambio climático y la migración, pueden tener un gran impacto en su expansión geográfica. El surgimiento, el resurgimiento y la rápida propagación de este virus son hoy en día un problema mundial de salud pública debido a las consecuencias clínicas que esta infección puede generar en grupos vulnerables. Desde
2015, América Latina se ha convertido en el punto de atención debido a la alta frecuencia de infecciones por ZIKV, a causa de su condición tropical (3).

Se han hecho esfuerzos para prevenir, detectar y responder a la infección, desarrollándose varios métodos para la detección del ZIKV. Estos métodos se pueden agrupar en ensayos inmunoenzimáticos ${ }^{(4)}$ como ELISA, aislamiento viral ${ }^{(5)}$ y métodos moleculares como la reacción en cadena de la polimerasa con transcriptasa inversa (RT-PCR) ${ }^{(6)}$. Sin embargo, cada

\footnotetext{
Centro Nacional de Salud Pública, Instituto Nacional de Salud. Lima, Perú

University of Miyazaki. Miyazaki, Japan.

Biólogo; ${ }^{b}$ tecnóloga médica; ${ }^{c}$ médico infectólogo,

Recibido: 18/09/2018 Aprobado: 24/07/2019 En línea: 13/08/2019
}

Citar como: Escalante-Maldonado O, Gustavo Gavilán R, García MP, Marcelo A, Pacheco E, Cabezas C, et al. Desarrollo y validación del método de amplificación isotérmica mediada en lazo para la detección del virus Zika. Rev Peru Med Exp Salud Publica. 2019;36(3):442-7. doi:http://dx.doi.org/10.17843/rpmesp.2019.363.3941. 
uno de estos métodos presenta desventajas tales como baja especificidad/sensibilidad (ELISA), alta complejidad y tiempo (aislamiento viral), así como elevados costos (RT-PCR) ${ }^{(7)}$. Además, todos estos métodos requieren condiciones especiales de laboratorio, dificultando su utilidad en la mayoría de las regiones donde prevalece la infección por ZIKV. Por tanto, es necesario encontrar una mejor alternativa, que incluya plataformas $y$ tecnologías de diagnóstico de mayor accesibilidad que puedan identificar de manera rápida, precisa y sencilla enfermedades infecciosas emergentes en el punto de atención, así como contribuir a la detección y vigilancia.

La amplificación isotérmica mediada en lazo, LAMP (por las siglas en inglés de Loop-mediated isothermal amplification) permite la detección en un solo paso de la amplificación génica a una sola temperatura ${ }^{\left({ }^{8}\right)}$ y se ha informado que LAMP es más simple y sensible que los métodos tradicionales de PCR convencionales. Últimamente, el método LAMP se ha utilizado en un formato de retro transcriptasa (RT-LAMP) para detectar virus ARN en un solo paso ${ }^{(9)}$. Además, el reactivo LAMP liofilizado no requiere condiciones especiales durante el almacenamiento, el transporte y la operación, por lo que puede usarse para detectar microorganismos patógenos incluso en países tropicales ${ }^{(10)}$. Si el reactivo LAMP liofilizado es aplicable a la detección del ZIKV, sería muy útil en varias partes del mundo.

En este estudio se desarrolló y validó, usando muestras de suero, un método específico, sensible y fácil de usar para detectar ZIKV basado en RT-LAMP, que puede realizarse en cualquier parte del mundo requiriendo condiciones mínimas de laboratorio.

\section{EL ESTUDIO}

\section{MUESTRAS DE SUERO}

Se emplearon muestras de suero de diversas regiones del Perú, colectadas bajo el Sistema Nacional de Vigilancia del Laboratorio de Referencia Nacional de Metaxénicas Virales (LRNMV) del Instituto Nacional de Salud del Perú (INS). Estas muestras correspondían a pacientes con cuadros clínicos febriles agudos. El tratamiento de los pacientes y las muestras se dieron de acuerdo a lo estipulado en el Protocolo de Investigación aprobado por el Comité Institucional de Ética en Investigación del INS.

\section{IDENTIFICACIÓN DEL VIRUS}

El LRNMV detectó ZIKV y otros arbovirus utilizando el método estándar de oro de reacción en cadena de la polimerasa con transcriptasa inversa (qRT-PCR), previamente descritos para Zika (11), Dengue (12),

\section{MENSAJES CLAVE}

Motivación para realizar el estudio. La introducción del virus Zika en Perú resalta la necesidad de realizar pruebas de diagnóstico en punto de atención, o cerca de la ubicación del paciente.

Principales hallazgos. Se desarrolló un método de diagnóstico de bajo costo, alto rendimiento, viabilidad y confiabilidad para el diagnóstico rápido de Zika.

Implicancias. El método de diagnóstico propuesto podría ser una alternativa de gran utilidad en instalaciones de atención primaria de salud en Perú.

Chikungunya ${ }^{(13)}$, fiebre amarilla ${ }^{(14)}$, Oropouche ${ }^{(15)} \mathrm{y}$ Mayaro ${ }^{(16)}$, y adaptados a los protocolos del INS.

\section{DISEÑO DE LOS PRIMERS}

Los nuevos primers ZIKV RT-LAMP se diseñaron basándose en la región del gen codificante de la enzima ARN polimerasa dependiente de ARN denominada NS5 (proteína no estructural 5) del genoma del ZIKV utilizando el software Primer Explorer V4 (Fujitsu System Solutions Ltd., Tokio, Japón). Las secuencias de nucleótidos conservadas dentro de la región NS5 se identificaron usando una alineación múltiple de 64 genomas completos del ZIKV disponibles de las bases de datos DDBJ / EMBL / GenBank (Tabla 1).

\section{EXTRACCIÓN DE ARN}

La extracción y purificación del ARN se realizaron utilizando el estuche comercial de purificación de ADN I ARN genómico GeneJET (Thermo Scientific, EE. UU.) de acuerdo con las instrucciones del fabricante. Los pasos opcionales o recomendados en el protocolo para eliminar los inhibidores de la reacción fueron seguidos.

Tabla 1. Nuevos primers para la detección del virus Zika por el método de amplificación isotérmica mediada en lazo de transcriptasa inversa (ZIKV RT-LAMP)

\begin{tabular}{ll}
\hline Primer & Secuencia 5' a 3' \\
\hline ZikV_F3 & GAGATGTACTGGGTCTCTGG \\
ZikV_B3 & GCTTCTCAGCGCGGAT \\
ZikV_FIP & TTCACTGGCCTCCTAGGCCCACCATAAAAAGTGTGTCCACC \\
ZikV_BIP & ATGTGAATCTCGGCTCTGGCAGCGGTTACCAATGATCTTCATG \\
ZikV_LF & GCCCCAAGAGGAGCTG \\
ZikV_LB & CGCGGGCTGTGGTAAGCTG \\
\hline
\end{tabular}


El ARN se usó inmediatamente en la reacción de RTLAMP o se almacenó en alícuotas a $-80^{\circ} \mathrm{C}$ hasta su uso.

\section{ENSAYO RT-LAMP UTILIZANDO REACTIVO LIOFILIZADO}

Los microtubos que contienen el reactivo liofilizado fueron tomados de un estuche comercialmente disponible que se dirige al ácido nucleico mediante el método LAMP, el reactivo de amplificación de ARN/ADN Loopamp D (Eiken Chemical Co., Ltd., Tokio, Japón). Los microtubos fueron transportados, almacenados y rehidratados a temperatura ambiente. La reacción de RT-LAMP se llevó a cabo en un tubo de $0,2 \mathrm{ml}$, con un volumen de reacción total de $30 \mu \mathrm{L}$, $5 \mu \mathrm{L}$ de solución de ARN, 1,3 $\mu \mathrm{L}$ de oligonucleotidos ZIKV RT-LAMP que contenían oligonucleótidos FIP y BIP (40 pmol), F y B (20 pmol), oligonucleotidos externos F3 y B3 (5 pmol), y $23,7 \mu \mathrm{L}$ de agua destilada. La tapa se aseguró firmemente y el microtubo se invirtió durante 3 minutos para rehidratar el reactivo que se encuentra en la tapa del tubo. El tubo se calentó a $65^{\circ} \mathrm{C}$ durante 1 h y a $80^{\circ} \mathrm{C}$ durante 5 min (inactivación enzimática). Los resultados de la reacción se determinaron visualmente por el cambio de color de marrón a verde en la solución de reacción. Considerándose negativo el tubo control en donde no se evidenció cambio de coloración.

\section{PRUEBA PRELIMINAR}

Se compararon siete muestras de suero que fueron previamente confirmadas como positivas para los siguientes agentes virales: ZIKV-1 $(n=2)$, DENV-1 $(n=1)$, DENV-2 $(n=1)$, DENV-3 $(n=1)$, DENV-4 $(n=1)$ y CHIKV $(n=1)$ usando las metodologías de qRT-PCR descritas para los virus mencionados. Luego se probó todas las muestras usando el ZIKV RT-LAMP para evaluar la especificidad. Además, una de las soluciones de ARN de ZIKV fue cuantificada y diluida en serie (10 veces) hasta 15 tubos. Luego, dichas diluciones fueron probadas por el ZIKV RT-LAMP y la qRT-PCR para evaluar la sensibilidad.

\section{EXPERIMENTO DE VALIDACIÓN DIAGNÓSTICA}

Se usaron un total de 300 muestras de suero para evaluar el rendimiento del ZIKV RT-LAMP. La mitad de ellos fueron muestras positivas de Zika. Las muestras de suero analizadas fueron recolectadas durante 2016-2017 en las regiones de Piura, Tumbes, Loreto, San Martín, Huánuco, Ucayali, Lima y Cajamarca. Las muestras se volvieron a evaluar primero mediante el método estándar de oro qRTPCR. Finalmente, las muestras de suero fueron probadas usando el método ZIKV RT-LAMP como se describió anteriormente y los resultados fueron comparados.

\section{ANÁLISIS ESTADÍSTICO}

Con el fin de determinar el desempeño del nuevo método ZIKV RT-LAMP, se evaluó la sensibilidad diagnóstica, la especificidad, el valor predictivo positivo y el valor predictivo negativo.

\section{HALLAZGOS}

\section{EVALUACIÓN INICIAL DE ZIK RT-LAMP}

Los nuevos primers ZIKV RT-LAMP se evaluaron con las 7 muestras de suero descritas anteriormente. Los resultados se evidenciaron a simple vista (Figura 1A), notando que sólo los resultados positivos de la muestra para ZIKV mediante los métodos estándar de oro qRT-PCR fueron positivos con ZIKV RT-LAMP. Además, una solución de ARN de ZIKV fue cuantificada obteniendo un valor inicial de $7 \mathrm{ng}$, para luego ser diluida en serie y analizada. El resultado de las pruebas del ZIKV RT-LAMP se determinó también de manera visual (Figura 1B).

Los resultados comparativos mostraron que el ZIKV RTLAMP es 1000 veces más sensible que RT-PCR en tiempo real, revelando un límite de detección tan bajo como 0,0007 pg (Tabla 2).

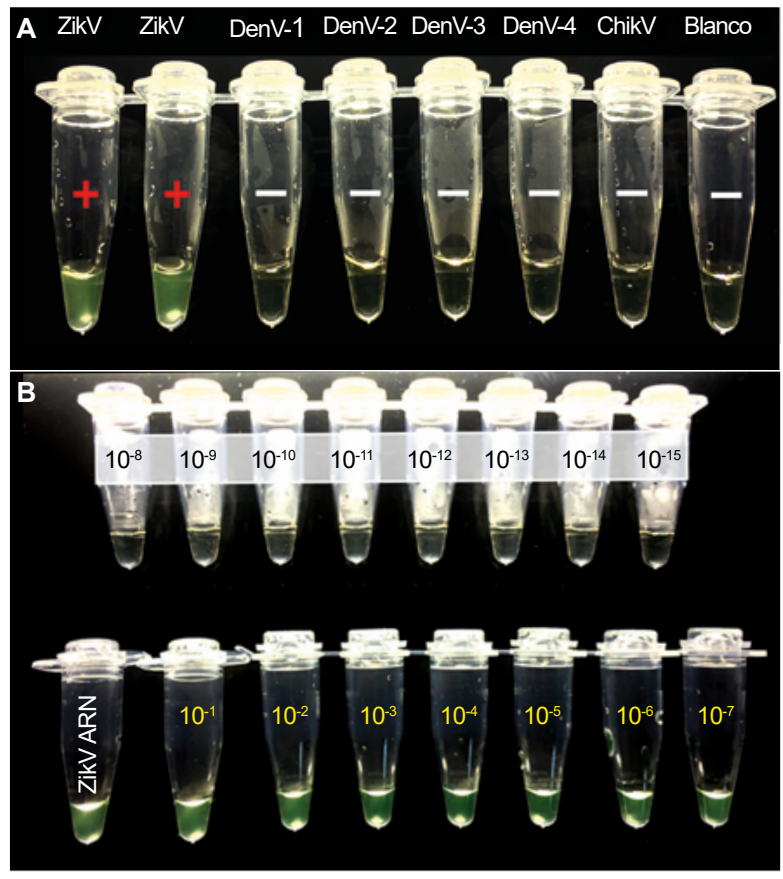

Figura 1. A. Especificidad del método para la detección del virus Zika por amplificación isotérmica mediada en lazo de transcriptasa inversa (ZIKV RT-LAMP), soluciones de ARN de ZIKV, DENV-1, DENV-2, DENV-3, DENV-4 y CHIKV reacción positiva (+) y negativa (+) LAMP juzgada visualmente. B. Sensibilidad del método ZIKV RT-LAMP, solución de ARN de ZIKV seguido por una serie de diluciones 10 veces 
Tabla 2. Comparación del límite de detección del virus Zika por el método de amplificación isotérmica mediada en lazo de transcriptasa inversa ZIKV RT-LAMP) y reacción en cadena de la polimerasa con transcriptasa inversa (qRT-PCR)

\begin{tabular}{lcc}
\hline $\begin{array}{l}\text { Cantidad de ARN } \\
\text { (picogramos) }\end{array}$ & RT-LAMP & qRT-PCR \\
\hline 7000 & + & + \\
700 & + & + \\
70 & + & + \\
7 & + & + \\
0,7 & + & + \\
0,07 & + & - \\
0,007 & + & - \\
0,0007 & + & - \\
0,00007 & - & - \\
\hline
\end{tabular}

El método ZIKV RT-LAMP fue 1000 veces más sensible que el qRT-PCR

\section{VALIDACIÓN DE LABORATORIO DE ZIKV RT-LAMP}

Se analizaron 300 muestras de suero mediante los métodos estándar de oro qRT-PCR y se determinó que 150 resultaron positivos a ZIKV, 15 a DENV-1, 15 a DENV-2, 15 a DENV-3, 15 a DENV-4, 20 a CHIKV, 10 a Fiebre Amarilla, 10 a Oropouche, 10 a Mayaro y 40 fueron negativos a los anteriores. Después, todas las muestras fueron analizadas con el método ZIKV RT-LAMP. Los resultados usando ambos métodos coincidieron perfectamente, excepto para una muestra que se reportó como positiva para ZIKV por el método estándar de oro qRT-PCR y negativa por el método ZIKV RT-LAMP (Tabla 3). Finalmente, se calculó la competencia del método ZIKV RT-LAMP con una sensibilidad del 99,3\% (IC 95\%: 97,7 - 100,0), una especificidad del 100\% (IC 95\%: 99,7 - 100,0), un valor predictivo positivo del 100\% (IC 95\%: 99,7 -100,0) y un valor predictivo negativo del 99,3\% (IC 95\%: 97,7 - 100,0).

\section{DISCUSIÓN}

Durante los últimos años, se han desarrollado varios métodos de amplificación de ARN para detectar ZIKV, como RT-PCR, SYBR Green en tiempo real RT-PCR y Taqman en qRT-PCR ${ }^{(7,11)}$. Aunque estos métodos han demostrado ser muy confiables, requieren instalaciones de laboratorio estandarizadas, equipos sofisticados e instrumentos especiales. Además, se necesita personal bien capacitado para ejecutar los experimentos. Este estudio presenta un método novedoso y altamente específico de RT-LAMP para la detección de ZIKV. Este método proporciona ventajas con respecto al tiempo, la operación, la viabilidad y el costo. Algunos grupos de investigación han desarrollado antes métodos basados en RT-LAMP para detectar ZIKV mostrando muy buenos resultados.

Calvert et al. reportaron una RT-LAMP con un nivel de sensibilidad 10 veces mayor que qRT-PCR ${ }^{(17)}$. No obstante, el método ZIKV RT-LAMP mostró un nivel de sensibilidad 1000 veces mayor que qRT-PCR. A diferencia de Wang et al. que reportaron un RT-LAMP probado en muestras clínicas simuladas mostrando una sensibilidad similar a qRT-PCR ${ }^{(18)}$, nuestra ZIKV RTLAMP se probó usando 300 muestras clínicas, 150 de ellas fueron ZIKV.

Korosaki et al. informaron sobre otro RT-LAMP que muestra resultados muy interesantes, alta sensibilidad,

Tabla 3. Validación del método para la detección del virus Zika por amplificación isotérmica mediada en lazo de transcriptasa inversa (ZIKV RT-LAMP) utilizando muestras de otros arbovirus

\begin{tabular}{lccccc}
\hline & \multicolumn{5}{c}{ ZIKV RT-LAMP vs qRT-PCR } \\
\cline { 2 - 6 } Especie* $^{*}$ Cantidad & $\begin{array}{c}\text { Verdaderos } \\
\text { positivos }\end{array}$ & $\begin{array}{c}\text { Falsos } \\
\text { positivos }\end{array}$ & $\begin{array}{c}\text { Verdaderos } \\
\text { negativos }\end{array}$ & $\begin{array}{c}\text { Falsos } \\
\text { negativos }\end{array}$ \\
\hline Zika & 150 & 149 & 0 & 0 & 1 \\
Dengue-1 & 15 & - & 0 & 15 & - \\
Dengue-2 & 15 & - & 0 & 15 & - \\
Dengue-3 & 15 & - & 0 & 15 & - \\
Dengue-4 & 15 & - & 0 & 15 & - \\
Chikungunya & 20 & - & 0 & 20 & - \\
Fiebre amarilla & 10 & - & 0 & 10 & - \\
Oropouche & 10 & - & 0 & 10 & - \\
Mayaro & 10 & - & 0 & 10 & - \\
Negativo a los anteriores & 40 & - & 0 & 40 & - \\
Total & 300 & 149 & 0 & 150 & 1 \\
\hline
\end{tabular}

*La identificación de la especie fue previamente realizada por el método estándar de oro de reacción en cadena de la polimerasa con transcriptasa inversa (qRT-PCR)

Guión: no aplica dato 
alta especificidad y se analizó utilizando muestras de suero, plasma y orina ${ }^{(19)}$. Sin embargo, el uso de reactivo líquido LAMP en la metodología puede ser muy problemático al momento de transportar y almacenar. En el ZIKV RT-LAMP se solucionó este problema al introducir el uso del reactivo LAMP liofilizado. Song et al. reportaron un método RT-LAMP que es simple, fácil de usar, rentable y aplicable en punto de atención, o cerca de la ubicación del paciente ${ }^{(20)}$. Parece tener la misma dirección que nuestro método, como la detección visual sin necesidad de equipo.

La ventaja del método ZIKV RT-LAMP es que ha probado también ser útil con muestras de suero obteniendo valores altos de sensibilidad y especificidad. Dichos valores responden principalmente al buen diseño de los primers y a la apropiada aplicación de la metodología. La única limitante es que la metodología LAMP no permite el diagnóstico diferencial en la misma reacción, aunque esto podría solucionarse diseñando primers para otros arbovirus y colocarlos en tubos adicionales.

En conclusión, el método ZIKV RT-LAMP desarrollado en este estudio permite una identificación rápida y confiable de ZIKV. Debido a su bajo costo, la practicidad y la operación simple pueden ser una buena alternativa para su utilización incluso en instalaciones de atención primaria de salud $u$ hospitales locales sin equipo de laboratorio complejo.

Contribución de los autores: EMO, CC, GGR, YW participaron en la concepción, delineación de hipótesis y diseño del estudio; EMO, GMP, MA, PE participaron en el análisis o interpretación de datos, redacción del artículo, EMO, CC, YW participaron en la revisión crítica del artículo.

Fuente de financiamiento: El estudio fue financiado por el Instituto Nacional de Salud del Perú.

Conflicto de interés: Todos los autores declaran que no tienen ningún conflicto de intereses en relación con esta publicación.

\section{REFERENCIAS BIBLIOGRÁFICAS}

1. Dick GWA. Zika virus. II. Pathogenicity and physical properties. Trans R Soc Trop Med Hyg. 1952;46(5):521-34.

2. Tsetsarkin KA, Chen R, Sherman MB, Weaver SC. Chikungunya virus: evolution and genetic determinants of emergence. Curr Opin Virol. 2011;1(4):310-7. doi: 10.1016/j.coviro.2011.07.004.

3. Ferguson NM, Cucunubá ZM, Dorigatti I, Nedjati-Gilani GL, Donnelly CA, Basáñez M-G, et al. Countering the Zika epidemic in Latin America. Science. 2016;353(6297):353-4. doi: 10.1126/ science.aag0219.

4. Kuno G. Serodiagnosis of flaviviral infections and vaccinations in humans. Adv Virus Res. 2003;61:3-65.

5. Alera MT, Hermann L, Tac-An IA, Klungthong C, Rutvisuttinunt W, Manasatienkij W, et al. Zika virus infection, Philippines, 2012. Emerg Infect Dis. 2015;21(4):722-4. doi: 10.3201/eid2104.141707.

6. Waggoner JJ, Gresh L, Mohamed-Hadley A, Ballesteros G, Davila MJV, Tellez Y, et al. Single-Reaction Multiplex Reverse Transcription PCR for Detection of Zika, Chikungunya, and Dengue Viruses. Emerg Infect Dis. 2016;22(7):1295-7. doi: 10.3201/ eid2207.160326.

7. Waggoner JJ, Pinsky BA. Zika Virus: Diagnostics for an Emerging
Pandemic Threat. J Clin Microbiol. 2016;54(4):860-7. doi: 10.1128/ JCM.00279-16.

8. Notomi T, Okayama H, Masubuchi $\mathrm{H}$, Yonekawa $\mathrm{T}$, Watanabe $\mathrm{K}$, Amino $\mathrm{N}$, et al. Loop-mediated isothermal amplification of DNA. Nucleic Acids Res. 2000;28(12):e63.

9. Teoh BT, Sam SS, Tan KK, Johari J, Danlami MB, Hooi P-S, et al. Detection of dengue viruses using reverse transcription-loop-mediated isothermal amplification. BMC Infect Dis. 2013;13(1):387. doi: 10.1186/14712334-13-387.

10. Escalante-Maldonado O, Kayali AY, Yamazaki W, Vuddhakul V, Nakaguchi Y, Nishibuchi M. Improvement of the quantitation method for the $\mathrm{tdh}+\mathrm{Vibrio}$ parahaemolyticus in molluscan shellfish based on most-probable- number, immunomagnetic separation, and loopmediated isothermal amplification. Front Microbiol. 2015;6:270. doi: 10.3389/ fmicb.2015.00270. eCollection 2015. /

11. Lanciotti RS, Kosoy OL, Laven JJ, Velez JO, Lambert AJ, Johnson AJ, et al. Genetic and Serologic Properties of Zika Virus Associated with an Epidemic, Yap State, Micronesia, 2007. Emerg Infect Dis. 2008;14(8):1232-9. doi: 10.3201/ eid1408.080287.
12. Johnson BW, Russell BJ, Lanciotti RS. Serotype-Specific Detection of Dengue Viruses in a Fourplex Real-Time Reverse Transcriptase PCR Assay. J Clin Microbiol. 2005;43(10):4977-83.

13. Johnson BW, Russell BJ, Goodman $\mathrm{CH}$. Laboratory Diagnosis of Chikungunya Virus Infections and Commercial Sources for Diagnostic Assays. J Infect Dis. 2016;214(suppl_5):S471-4.

14. Domingo C, Patel P, Yillah J, Weidmann M, Méndez JA, Nakouné ER, et al. Advanced yellow fever virus genome detection in point-of-care facilities and reference laboratories. J Clin Microbiol. 2012;50(12):4054-60. doi: 10.1128/JCM.01799-12.

15. García MP, Merino NS, Figueroa D, Marcelo A, Tineo V. E, Manrique C, et al. Detección de la circulación del virus Oropuche en la región Madre de Dios, Perú (diciembre 2015 - enero 2016) Rev Peru Med Exp Salud Publica. 2016;33(2):380-1.

16. Friedrich-Jänicke B, Emmerich P, Tappe D, Günther S, Cadar D, SchmidtChanasit J. Genome Analysis of Mayaro Virus Imported to Germany from French Guiana. Emerg Infect Dis. 2014;20(7):1255-7. doi: 10.3201/ eid2007.140043.

17. Calvert AE, Biggerstaff BJ, Tanner NA, Lauterbach $M$, Lanciotti RS Rapid colorimetric detection of Zika 
virus from serum and urine specimens by reverse transcription loop-mediated isothermal amplification (RT-LAMP). PLoS One. 2017;12(9):e0185340. doi: 10.1371/journal.pone.0185340.

18. Wang X, Yin F, Bi Y, Cheng G, Li J, Hou $L$, et al. Rapid and sensitive detection of Zika virus by reverse transcription loopmediated isothermal amplification. J Virol Methods. 2016;238:86-93. doi: 10.1016/j.jviromet.2016.10.010.
19. Kurosaki Y, Martins DBG, Kimura M, Catena A dos S, Borba MACSM, Mattos S da S, et al. Development and evaluation of a rapid molecular diagnostic test for Zika virus infection by reverse transcription loop-mediated isothermal amplification. Sci Rep. 2017;7(1):13503. doi: 10.1038/s41598-017-13836-9.

20. Song J, Mauk MG, Hackett BA, Cherry S, Bau HH, Liu C. Instrument-Free Point-of-Care Molecular
Detection of Zika Virus. Anal Chem. 2016;88(14):7289-94. doi: 10.1021/ acs.analchem.6b01632.

\section{Correspondencia: Oscar Roberto Escalante Maldonado}

Dirección: Jr. Cápac Yupanqui 1400, Jesús Maria, Lima, Perú.

Teléfono: 511-7481111 anexo 2123

coreoelectrónico:oescalante@ins.gob.pe

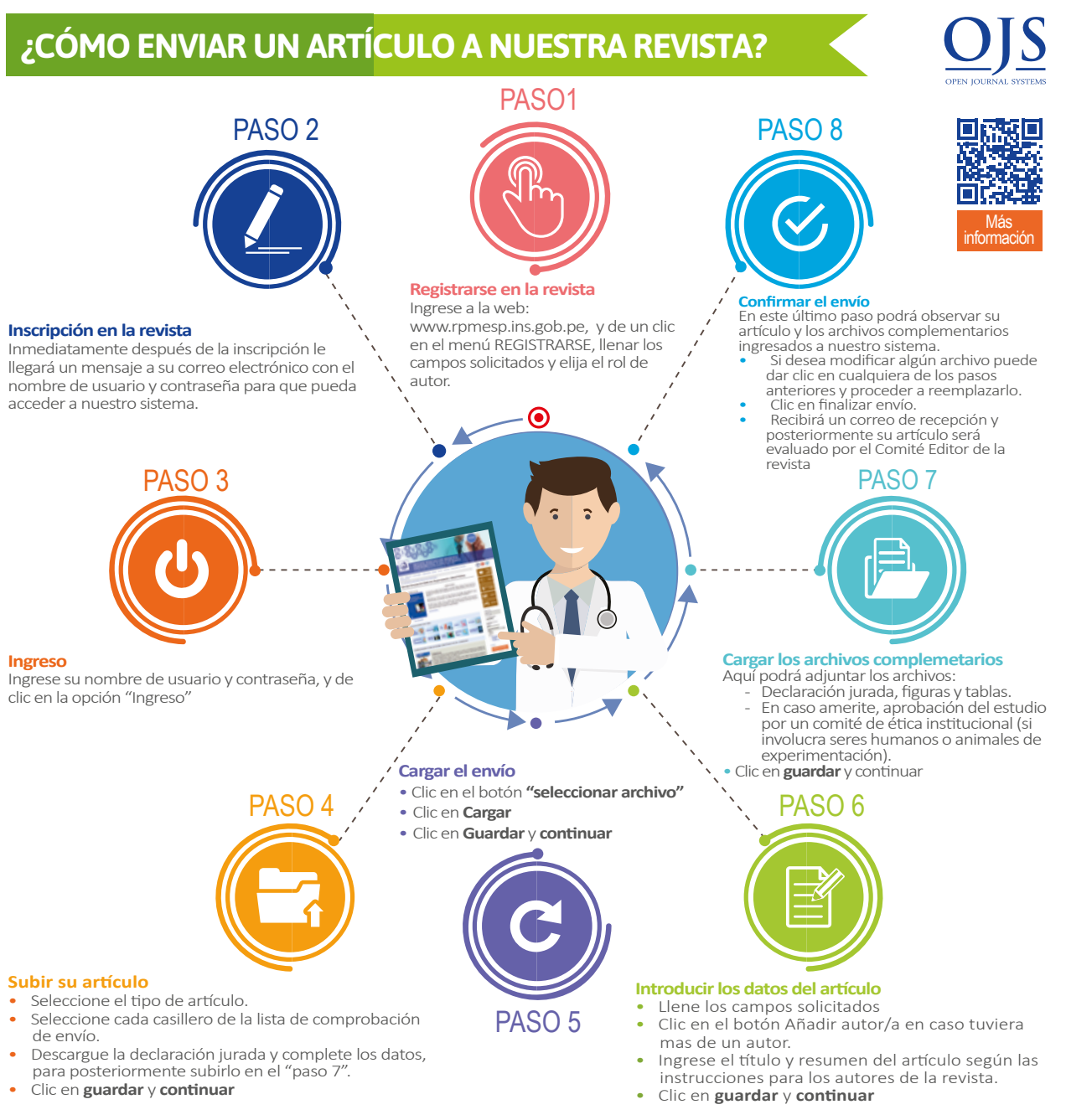

